\title{
THE CONSEQUENCES OF USING INADEQUATE TESTERS IN THE SIMPLIFIED TRIPLE TEST-CROSS
}

\author{
D. S. VIRK and J. L. JINKS \\ Department of Genetics, University of Birmingham, Birmingham B15 2TT
}

Received 22.x.76

\begin{abstract}
Summary
The genetical consequences of common alleles in the $L_{1}$ and $L_{2}$ testers of a simplified version of the triple test-cross which is applicable to populations of inbred lines are examined. The test for epistasis under these circumstances becomes ambiguous and can spuriously detect non-allelic interactions when they may not exist although it still provides a test for epistasis and the adequacy of the testers simultaneously. The tests of significance and the estimates of additive variation are biased to an extent related to the dominance and dominance $x$ additive effects of the common loci while the significance and estimates of dominance variation are deflated because they reflect the dominance effects at the non-common loci only. The covariance of sums and differences is also underestimated for the same reasons. These expectations are illustrated by analysing the 190 simplified triple test-crosses that could be extracted from a $20 \times 20$ diallel set of crosses between pure-breeding lines of Nicotiana rustica.
\end{abstract}

\section{INTRODUCTION}

Jinks, Perkins And Breese (1969) have described a simplified version of the triple test-cross of Kearsey and Jinks (1968) in which the $\mathrm{L}_{3}$ families (testcrosses to an $\mathrm{F}_{1}$ tester) are replaced by $\mathrm{P}$ families (selfs of the population under test). The use of this simplified version is restricted to populations of pure-breeding lines, so that the $\mathbf{P}$ families are the pure-breeding lines themselves, and the analysis yields unambiguous results only if the $\mathrm{L}_{1}$ and $\mathrm{L}_{2}$ pure-breeding testers differ at all the $k$ loci at which individuals in the population may differ.

If $\mathrm{L}_{1}$ and $\mathrm{L}_{2}$ differ at only $k-k_{1}=k_{2}$ loci where $k_{2}<k$ and $k_{1}$ is the number of loci at which both $\mathrm{L}_{1}$ and $\mathrm{L}_{2}$ are homozygous for the same allele, the tests and estimates of the additive, dominance and epistatic components are biased and the different kinds of gene action and interaction are confounded. In this paper theoretical expectations for these biases are derived and compared with experimental results obtained from Nicotiana rustica breeding programmes.

\section{THEORY}

Taking the simplest case of two loci and two alleles at each locus, $A, a$ and $B, b$ there are four possible pure-breeding lines $A A B B, A A b b, a a B B$ and $a a b b$ and these can be paired to yield 10 , or $2^{k}\left[2^{k}+1\right] / 2, \mathrm{~L}_{1}$ and $\mathrm{L}_{2}$ pairs of testers (table 1). Of these, four pairs have one gene in common $(3,4,5$ and 6) and four, both genes in common (7, 8, 9 and 10). The theoretical expectations of the 10 simplified triple test-crosses involving these pairs of testers to investigate a population of pure-breeding lines in linkage equilibrium for equal and unequal gene frequencies are summarised in tables 1 to 4 . 


\section{TABLE 1}

The expected $\sigma^{2}\left(L_{1 \mathbf{i}}+L_{21}-\bar{P}_{\mathbf{1}}\right)$ for the 10 tester combinations in populations of pure-breeding lines differing for two alleles with equal frequencies at two loci and in linkage equilibrium

Expectations

\begin{tabular}{|c|c|c|}
\hline $\begin{array}{l}\text { Tester } \\
\text { combination }\end{array}$ & $\begin{array}{l}\text { Epistasis } \\
\text { absent }\end{array}$ & Epistasis present \\
\hline 1. $\begin{aligned} \mathrm{L}_{1} & =A A B B \\
\mathrm{~L}_{2} & =a a b b\end{aligned}$ & 0 & $\frac{1}{9}\left[\frac{1}{4}\left(l_{a b}-i_{a b}\right)^{2}-\frac{1}{2}\left(j_{a b}-j_{b a}\right)^{2}\right]$ \\
\hline 2. $\begin{aligned} \mathrm{L}_{1} & =A A b b \\
\mathrm{~L}_{2} & =a a B B\end{aligned}$ & 0 & $\frac{1}{8}\left[\frac{1}{4}\left(l_{a b}+i_{a b}\right)^{2}+\frac{1}{2}\left(j_{a b}+j_{b a}\right)^{2}\right]$ \\
\hline 3. $\begin{aligned} \mathrm{L}_{1} & =A A B B \\
\mathrm{~L}_{2} & =A A b b\end{aligned}$ & $\frac{1}{9} h_{a}^{2}$ & $\frac{1}{9}\left[h_{a}^{2}+\frac{1}{4}\left(j_{a b}-l_{a b}\right)^{2}+\frac{1}{2}\left(j_{b a}+i_{a b}\right)^{2}+h_{a}\left(l_{a b}-j_{a b}\right)\right]$ \\
\hline 4. $\begin{aligned} \mathrm{L}_{1} & =A A B B \\
\mathrm{~L}_{2} & =a a B B\end{aligned}$ & $\frac{1}{9} h_{b}^{2}$ & $\frac{1}{9}\left[h_{b}^{2}+\frac{1}{4}\left(j_{b a}-l_{a b}\right)^{2}+\frac{1}{2}\left(j_{a b}+i_{a b}\right)^{2}+h_{b}\left(l_{a b}-j_{b a}\right)\right]$ \\
\hline 5. $\begin{aligned} \mathrm{L}_{1} & =a a B B \\
\mathrm{~L}_{2} & =a a b b\end{aligned}$ & $\frac{1}{9} h_{a}^{2}$ & $\frac{1}{9}\left[h_{a}^{2}+\frac{1}{4}\left(j_{a b}+l_{a b}\right)^{2}+\frac{1}{2}\left(j_{b a}-i_{a b}\right)^{2}+h_{a}\left(l_{a b}+j_{a b}\right)\right]$ \\
\hline 6. $\begin{aligned} \mathrm{L}_{1} & =A A b b \\
\mathrm{~L}_{2} & =a a b b\end{aligned}$ & $\frac{1}{9} h_{b}^{2}$ & $\frac{1}{9}\left[h_{b}^{2}+\frac{1}{4}\left(j_{b a}+l_{a b}\right)^{2}+\frac{1}{2}\left(j_{a b}-i_{a b}\right)^{2}+h_{b}\left(l_{a b}+j_{b a}\right)\right]$ \\
\hline 7. $\begin{aligned} \mathrm{L}_{1} & =A A B B \\
\mathrm{~L}_{2} & =A A B B\end{aligned}$ & $\frac{1}{9}\left[h_{a}^{2}+h_{b}^{2}\right]$ & $\begin{array}{l}\frac{1}{9}\left[h_{a}^{2}+h_{b}^{2}+\frac{3}{4}\left(l_{a b}^{2}+i_{a b}^{2}+j_{a b}^{2}+j_{b a}^{2}\right)+\left(h_{a}+h_{b}\right)\left(l_{a b}-i_{a b}\right)+\left(h_{a}-h_{b}\right)\left(j_{b a}-j_{a b}\right)\right. \\
\left.\quad+\frac{1}{2}\left(i_{a b}-l_{a b}\right)\left(j_{a b}+j_{b a}\right)-\frac{1}{2} j_{a b} j_{b a}-\frac{3}{2} l_{a b} i_{a b}\right]\end{array}$ \\
\hline 8. $\begin{aligned} \mathrm{L}_{1} & =a a b b \\
\mathrm{~L}_{2} & =a a b b\end{aligned}$ & $\frac{1}{a}\left[h_{a}^{2}+h_{b}^{2}\right]$ & $\begin{aligned} \frac{1}{9}\left[h_{a}^{2}+h_{b}^{2}+\frac{3}{(2}\left(l_{a b}^{2}+i_{a b}^{2}+j_{a b}^{2}+j_{b a}^{2}\right)\right. & +\left(h_{a}+h_{b}\right)\left(l_{a b}-i_{a b}\right)+\left(h_{a}+h_{b}\right)\left(j_{a b}+j_{b a}\right) \\
& \left.+\frac{1}{2}\left(i_{a b}-l_{a b}\right)\left(j_{a b}-j_{b a}\right)-\frac{1}{2} j_{a b} j_{b a}-\frac{3}{2} l_{a b} i_{a b}\right]\end{aligned}$ \\
\hline 9. $\begin{aligned} \mathrm{L}_{1} & =A A b b \\
\mathrm{~L}_{2} & =A A b b\end{aligned}$ & $\frac{1}{9}\left[h_{a}^{2}+h_{b}^{2}\right]$ & $\begin{array}{l}\frac{1}{9}\left[h_{a}^{2}+h_{b}^{2}+\frac{3}{4}\left(l_{a b}^{2}+i_{a b}^{2}+j_{a b}^{2}+j_{b a}^{2}\right)+\left(h_{a}+h_{b}\right)\left(i_{a b}+l_{a b}\right)-\left(h_{a}-h_{b}\right)\left(j_{a b}+j_{b a}\right)\right. \\
\left.\quad+\frac{1}{2}\left(i_{a b}-l_{a b}\right)\left(j_{a b}-j_{b a}\right)-\frac{1}{2} j_{a b} j_{b a}+\frac{3}{2} l_{a b} i_{a b}\right]\end{array}$ \\
\hline 10. $\begin{aligned} \mathrm{L}_{1} & =a a B B \\
\mathrm{~L}_{2} & =a a B B\end{aligned}$ & $\frac{1}{9}\left[h_{a}^{2}+h_{b}^{2}\right]$ & $\begin{array}{l}\frac{1}{9}\left[h_{a}^{2}+h_{b}^{2}+\frac{3}{4}\left(l_{a b}^{2}+i_{a b}^{2}+j_{a b}^{2}+j_{b a}^{2}\right)+\left(h_{a}+h_{b}\right)\left(i_{a b}+l_{a b}\right)+\left(h_{a}-h_{b}\right)\left(j_{a b}+j_{b a}\right)\right. \\
\left.\quad+\frac{1}{2}\left(i_{a b}+l_{a b}\right)\left(j_{a b}-j_{b a}\right)+\frac{1}{2} j_{a b} j_{b a}+\frac{3}{2} l_{a b} i_{a b}\right]\end{array}$ \\
\hline
\end{tabular}

\section{(i) Test for epistasis}

The test for epistasis of Jinks et al. (1969) is presented in table 1. Where the testers differ at both loci (pairs 1 and 2) the expected variance of $\overline{\mathrm{L}}_{1 i}+\mathrm{L}_{2 i}-\overline{\mathrm{P}}_{i}$ is zero in the absence of epistasis and it therefore provides a test for its presence. For the testers with alleles in common (pairs 3 to 10), however, it has an expectation which depends on the dominance effects at the $k_{1}$ common loci. It can therefore take a value even in the absence of epistasis and it is no longer a valid test of the presence of epistasis unless it can be shown that the testers differ at all relevant loci. In all circumstances, however, it is a test of the adequacy of a simple additive-dominance model which assumes that there is no epistasis and that the testers have no common alleles. The confounding of epistasis and dominance when the testers do not satisfy this requirement does not apply to the more general test for epistasis in the full triple test-cross analysis of Kearsey and Jinks (1968).

The generalised expectations for various genetical parameters when a simple model holds but $k_{1}>0$ are discussed below.

(ii) Estimation of additive and dominance components

The expectations for the variance of $\mathrm{L}_{1 i}+\mathrm{L}_{2 i}\left(\sigma_{s}^{2}\right)$ given in table 2 show that when the testers have alleles in common this is no longer an estimate 
TABLE 2

The expected variances of sums $\left(\sigma_{s}^{2}\right)$, differences $\left(\sigma_{d}^{2}\right)$ and their covariance for the 10 tester combinations for a digenic case without epistasis in linkage equilibrium

Tester combination

Coefficients

$(a)^{*}$

$(b) *$

1. $\mathrm{L}_{1}=A A B B$

$\mathrm{L}_{2}=a a b b$

2. $\mathrm{L}_{1}=A A b b$

$\mathrm{L}_{2}=a a B B$

3. $\mathrm{L}_{1}=A A B B$

$\mathrm{L}_{2}=A A b b$

4. $\mathrm{L}_{1}=A A B B$

$\mathrm{L}_{2}=a a B B$

5. $\mathrm{L}_{1}=a a B B$

$\mathrm{L}_{2}=a a b b$

6. $\mathrm{L}_{1}=A A b b$

$\mathrm{L}_{2}=a a b b$

7. $\mathrm{L}_{1}=A A B B$

$\mathrm{L}_{2}=A A B B$

8. $\mathrm{L}_{1}=a a b b$

$\mathrm{L}_{2}=a a b b$

9. $\mathrm{L}_{1}=A A b b$

$\mathrm{L}_{2}=A A b b$

10. $\mathrm{L}_{1}=a a B B$

$\mathrm{L}_{2}=a a B B$

Expectations

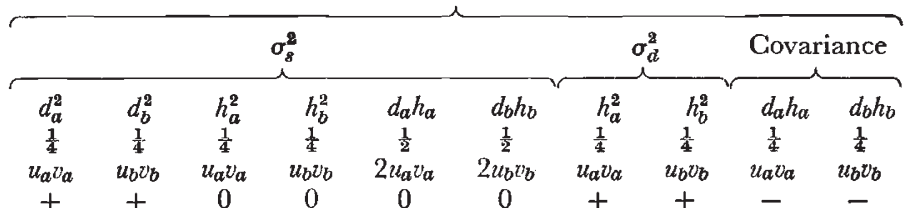

* (a) and (b) correspond to equal and unequal gene frequencies.

of the purely additive gene effects but contains the dominance and dominance $\times$ additive effects at the $k_{1}$ common loci. The sign of these cross product terms depends upon whether the common alleles are dominant (negative sign) or recessive (positive sign) irrespective of the direction of dominance.

The expectations may be generalised by using a coefficient of association (Mather and Jinks, 1971).

$$
r_{1}=\frac{\left(k_{1}-2 k_{1}^{\prime}\right)}{k_{1}}
$$

where, of the $k_{1}$ loci at which there are common alleles in the testers $k_{1}^{\prime}$ are decreasing and $\left(k_{1}-k_{1}^{\prime}\right)$ increasing alleles. The expectation of $\sigma_{s}^{2}$ for unequal gene frequencies then becomes

$$
\begin{aligned}
\sigma_{s}^{2} & =\sum_{i=1}^{k} u v d_{i}^{2}+\sum_{i=1}^{k_{1}} u v h_{i}^{2}-2 r_{1} \sum_{i=1}^{k_{1}} u v d_{i} h_{i} \\
& =\frac{1}{4} D_{k}+\frac{1}{4} H_{k_{1}}-\frac{1}{2} F_{k_{1}} .
\end{aligned}
$$

The expectations for the variance of $\bar{L}_{1 i}-\vec{L}_{2 i}\left(\sigma_{d}^{2}\right)$ are also given in table 2. While this depends solely on the dominance effects of the genes for all pairs of testers it reflects these effects at only the $k_{2}$ loci at which the 
testers differ. The generalised expectation for the variance of differences is therefore:

$$
\sigma_{d}^{2}=\sum_{i=1}^{k_{2}} u v h_{i}^{2}=\frac{1}{4} H_{k_{2}}
$$

\section{(iii) Covariance of sums and differences}

The covariance of $\overline{\mathrm{L}}_{1 i}+\overline{\mathrm{L}}_{2 i}$ and $\overline{\mathrm{L}}_{1 i}-\overline{\mathrm{L}}_{2 i}$ contains no contributions from the genes at the $k_{1}$ common loci while the contributions of the $k_{2}$ noncommon loci depend on their degree of association in the testers (table 2). If we write

$$
r_{2}=\frac{k_{2}-2 k_{2}^{\prime}}{k_{2}}
$$

where $k_{2}^{\prime}$ of the $k_{2}$ non-common alleles with increasing effect are present along with $k_{2}-k_{2}^{\prime}$ alleles of decreasing effect in $\mathrm{L}_{1}$ and vice versa in $\mathrm{L}_{2}$ (see Mather and Jinks, 1971) then the expectation is

$$
\operatorname{cov}_{\mathrm{sums} / \mathrm{diffs}}=-r_{2} \sum_{i=1}^{k_{2}} u v d_{i} h_{i}=-\frac{1}{4} F_{k_{2}} \text {. }
$$

The theoretical expectations for the variance of sums, differences and their covariance in the presence of digenic interactions are given in tables 3 and 4.

\section{RESUlts AND INTERPRETATION}

From the $20 \times 20$ diallel set of crosses between pure-breeding lines derived by selfing from the cross between varieties 1 and 5 of Nicotiana rustica, carried out by Dr E. L. Breese at Birmingham (experiment 1 of Jinks et al., 1969), 190 pairs of testers and sets of triple test-crosses can be extracted. One of these pairs (number 82) consists of lines 5 and 7 which are the extreme phenotypes and hence probably differ at more loci than any other pair of testers. The analyses of the simplified triple test-cross using these as testers are presentcd in table 5 . The 40 degrees of freedom for the error variance $\left(\sigma_{e}^{2}\right)$ have been obtained by pooling the blocks $\times$ families $\times$ reciprocals $(19)$, blocks $\times$ reciprocals (1), bctween reciprocals (1) and families $\times$ reciprocals (19) (see Jinks, 1956 and Jinks et al., 1969). There is no evidence of epistasis or of common genes in the testers for either flowering time or final height (table 5) hence an additive-dominance model is adequate. There is partial dominance in the direction of the early flowering while the low level of dominance for plant height is ambidirectional.

The results from the other 189 pairs should deviate from those for pair 82 solely because of the biases caused by common alleles in $\mathrm{L}_{1}$ and $\mathrm{L}_{2}$. To illustrate the theoretical and observed distribution of these biases the estimates of the genetical components for each of the 190 pairs of testers have been plotted against a measure of the phenotypic difference between each pair, namely, $[d]=\frac{1}{2}\left[\vec{L}_{1}-\bar{L}_{2}\right]=r_{2} \sum_{i=1}^{k_{2}} d_{i}$. Since, however, the genetical components are variances the axes have been made more comparable by plotting them against $[d]^{2}$. The cxpected relationships assuming an 
SIMPLIFIED TRIPLE TEST-CROSS

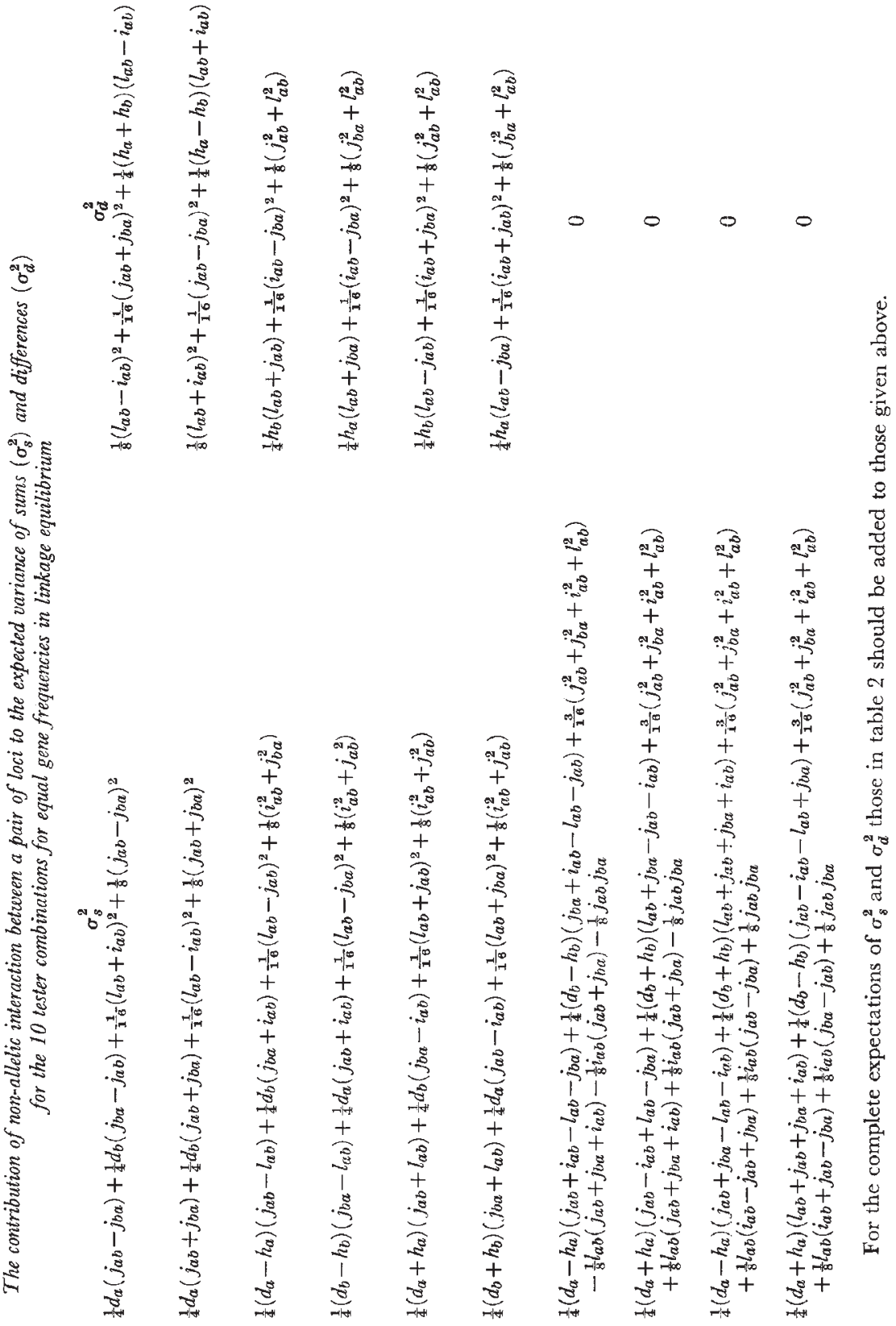

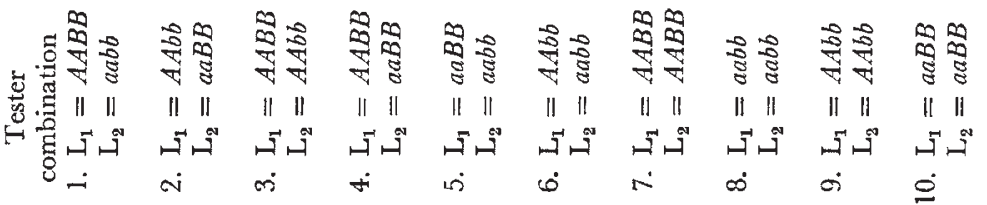
$38 / 2-\mathrm{H}$ 


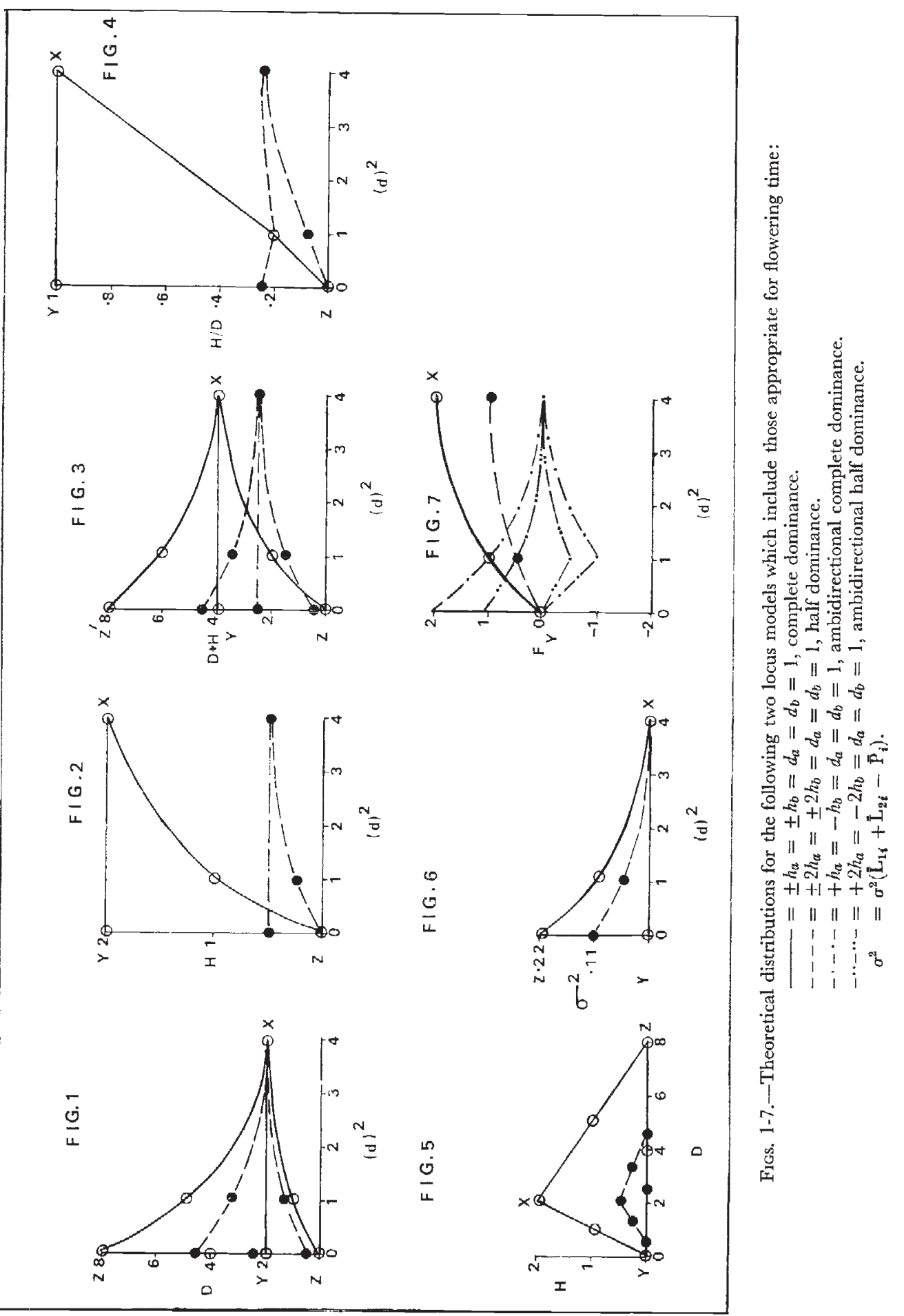



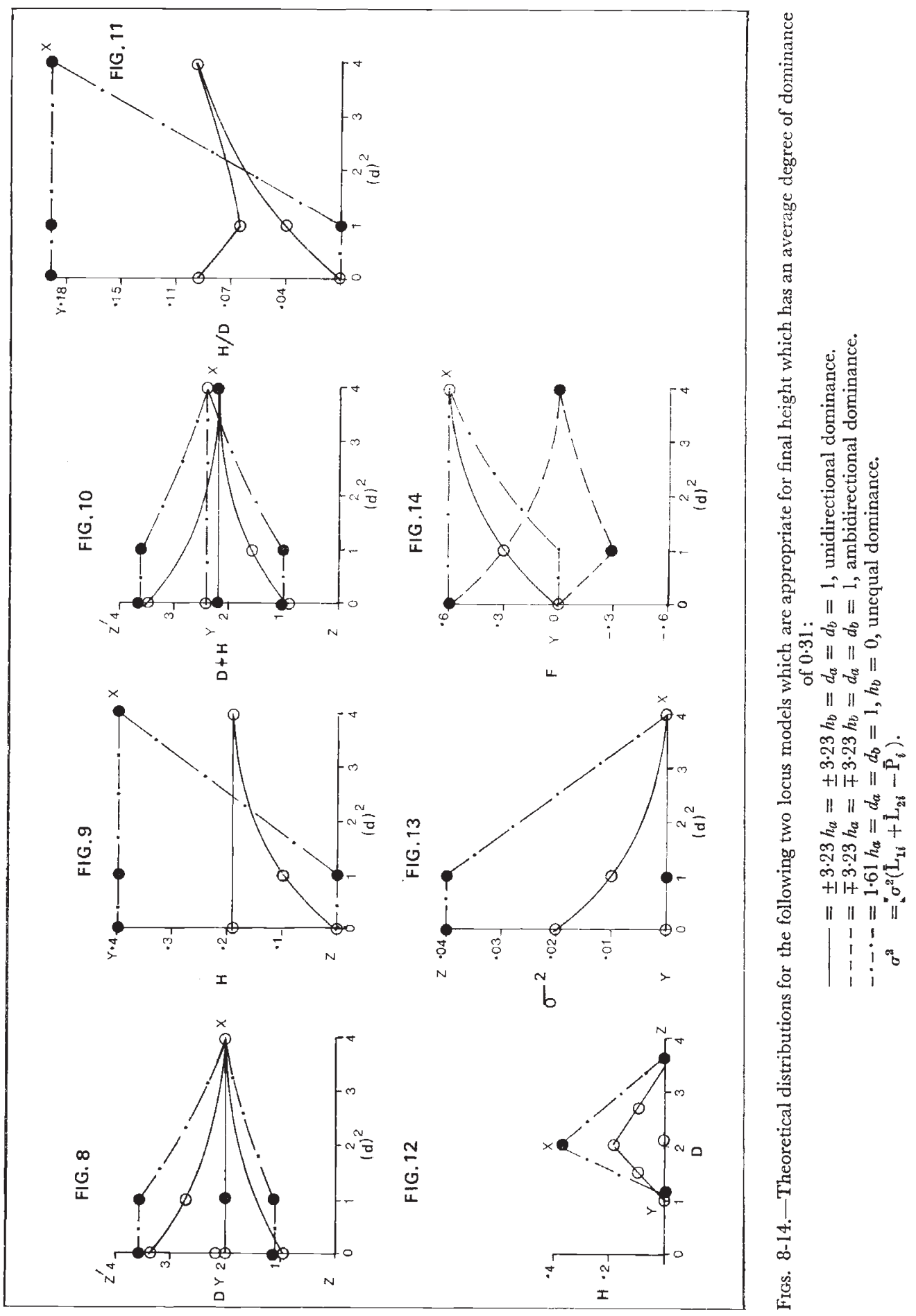


\section{TABLE 4}

The contributions of non-allelic interactions between a pair of loci to the expected covariance between $L_{1 i}+L_{2 i}$ and $L_{1 i}-L_{2 i}$ for the 10 tester combinations for equal gene frequencies in linkage equilibrium

Tester

combination

Expectations of covariance

1. $\mathrm{L}_{1}=A A B B \quad \frac{1}{8}\left(d_{a}+d_{b}\right)\left(i_{a b}-l_{a b}\right)+\frac{1}{8}\left(h_{a}-h_{b}\right)\left(j_{b a}-j_{a b}\right)-\frac{1}{1} \bar{b}\left(j_{a b}+j_{b a}\right)\left(i_{a b}+l_{a b}\right)$

$\mathrm{L}_{2}=a a b b$

2. $\mathrm{L}_{1}=A A b b \quad \frac{1}{8}\left(d_{b}-d_{a}\right)\left(i_{a b}+l_{a b}\right)+\frac{1}{8}\left(h_{b}-h_{a}\right)\left(j_{a b}+j_{b a}\right)+\frac{1}{18}\left(j_{a b}-j_{b a}\right)\left(i_{a b}+l_{a b}\right)$

$\mathrm{L}_{2}=a a B B$

3. $\mathrm{L}_{1}=A A B B \quad \frac{1}{8}\left(d_{a}-h_{a}\right)\left(i_{a b}-j_{b a}\right)-\frac{1}{8} d_{b}\left(l_{a b}+j_{a b}\right)-\frac{1}{8} h_{b}\left(i_{a b}+j_{b a}\right)-\frac{1}{16}\left(i_{a b}+j_{b a}\right)\left(l_{a b}+j_{a b}\right)$

$\mathrm{L}_{2}=A A b b$

4. $\mathrm{L}_{1}=A A B B \quad \frac{1}{8}\left(d_{b}-h_{b}\right)\left(i_{a b}-j_{a b}\right)-\frac{1}{8} d_{a}\left(l_{a b}+j_{b a}\right)-\frac{1}{8} h_{a}\left(i_{a b}+j_{a b}\right)-\frac{1}{16}\left(i_{a b}+j_{a b}\right)\left(l_{a b}+j_{b a}\right)$

$L_{2}=a a B B$

5. $\mathrm{L}_{1}=a a B B \quad \frac{1}{8}\left(d_{a}+h_{a}\right)\left(i_{a b}+j_{b a}\right)-\frac{1}{8} d_{b}\left(l_{a b}-j_{a b}\right)+\frac{1}{8} h_{b}\left(i_{a b}-j_{b a}\right)+\frac{1}{1 \frac{1}{6}}\left(i_{a b}-j_{b a}\right)\left(l_{a b}-j_{a b}\right)$

$\mathrm{L}_{2}=a a b b$

6. $L_{1}=A A b b \quad \frac{1}{8}\left(d_{b}+h_{b}\right)\left(i_{a b}+j_{a b}\right)-\frac{1}{8} d_{a}\left(l_{a b}-j_{b a}\right)+\frac{1}{8} h_{a}\left(i_{a b}-j_{a b}\right)+\frac{1}{16}\left(i_{a b}-j_{a b}\right)\left(l_{a b}-j_{b a}\right)$

$\mathrm{L}_{2}=a a b b$

7. $\mathrm{L}_{1}=A A B B \quad 0$

$\mathrm{L}_{2}=A A B B$

8. $\mathrm{L}_{1}=a a b b$

$\mathrm{L}_{\mathbf{2}}=a a b b$

9. $\mathrm{L}_{1}=A A b b$

$\mathrm{L}_{2}=A A b b$

10. $\mathrm{L}_{1_{1}}=a a B B$

$\mathrm{L}_{2}=a a B B$

For the complete expectations of the covariance those in table 2 should be added to those given above.

\section{TABLE 5}

Analyses of variance for additive (sums), dominance (differences) and epistasis for the phenotypic extreme pair of testers, inbreds 5 and $17 \dagger$

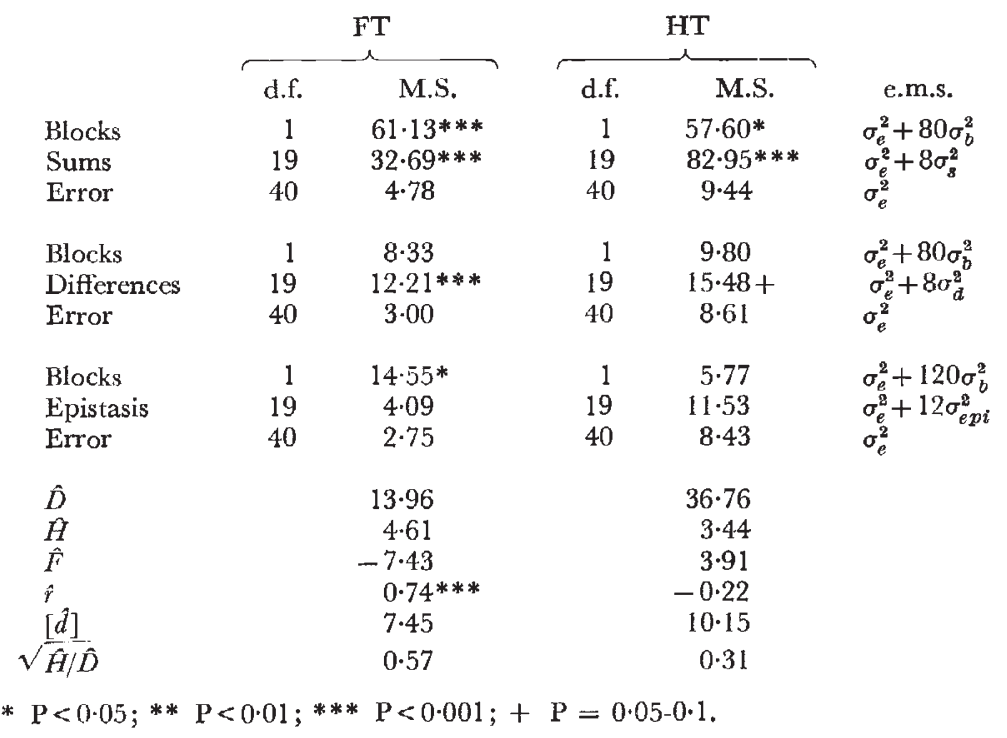

$\dagger$ Reciprocal crosses have been used as the only true replicates in these analyses and they differ in detail from those presented by Jinks et al. (1969). 
additive-dominance model for two loci at which $h_{a}=h_{b}=d_{a}=d_{b}=1$, $2 h_{a}=2 h_{b}=d_{a}=d_{b}=1$ and $3 \cdot 23 h_{a}=3 \cdot 23 h_{b}=d_{a}=d_{b}=1$ for unidirectional and ambidirectional dominance, respectively and $1.61 h_{a}=d_{a}=$ $d_{b}=1\left(h_{b}=0\right)$ for unequal dominance effects at the two loci are presented in figs. 1-14. Flowering time, in general, showed the expected distributions for partial, unidirectional dominance (figs. 1-7) while final height showed the expected distributions for a low level of dominance with unequal dominance effects (figs. 8-14). The more important of these relationships are discussed below.

\section{(i) Relationship of $\mathrm{D}$ and $[\mathrm{d}]^{2}$}

The unbiased estimates of $D$ fall on the line joining $\mathrm{X}$ and $\mathrm{Y}$ in figs. $\mathrm{l}$ and 8 and these are obtained when $L_{1}$ and $L_{2}$ differ at all loci irrespective of whether the alleles are associated or dispersed. The biased estimates of $D$ resulting from common alleles in $\mathrm{L}_{1}$ and $\mathrm{L}_{2}$ occupy the areas XYZ and $\mathrm{XYZ}$ for the dominant and recessive common alleles, respectively, irrespective of the direction of dominance. The lower the level of dominance the smaller are these areas and with no dominance all estimates should fall on the line XY.

The biases in the maximum and minimum estimates of $D$ (figs. 15 and 19) which coincide with $[d]^{2} \bumpeq 0$ are 10.96 and -11.76 for flowering time, and 14.55 and -24.57 for final height. They are the joint effect of the $\frac{1}{4} \sum_{i=1}^{k_{1}} h_{i}^{2}-\frac{1}{2} r_{1} \sum_{i=1}^{k_{1}} d_{i} h_{i}$ contributions of the common genes but the cross product term can be separated by computing the deviation of $\hat{D}+\hat{H}$ from that of the associated pair (table 2). The minimum estimates of $D$ for both characters are significantly greater than zero which is expected for partial dominance (figs. 15 and 19). These observed distributions show 89 and 95 per cent of the points for flowering time and final height, respectively falling in the lower half which is expected if there are more dominant than recessive alleles in the tester pairs.

The ratio of $[d]^{2}$ and $D$ at point $\mathrm{X}$ (the completely associated pairs of testers) is an estimate of the number of effective factors $(k)$ since for this pair:

$$
\frac{[d]^{2}}{D}=\frac{\left[\sum_{i=1}^{k_{2}} d_{i}\right]^{2}}{\sum_{i=1}^{k_{2}} d_{i}^{2}}=\frac{k_{2}^{2} d^{2}}{k_{2} d^{2}\left(1+V_{\alpha}\right)}
$$

where $d$ is the mean and $V_{\alpha}$ is the variance of $d_{i}$ over the $k_{2}=k$ loci. If all $d$ 's are equal $V_{\alpha}=0$ and we have an estimate of $k$, otherwise it is, of course, an underestimate (see Mather and Jinks, 1971). The number of effective factors so estimated were 3.98 (4) and 2.80 (3) for flowering time and final height, respectively.

\section{(ii) Relationship between $\mathrm{H}$ and $[\mathrm{d}]^{2}$}

Figs. 2 and 9 show that common genes $\left(k_{1}>0\right)$ in the $\mathrm{L}_{1}$ and $\mathrm{L}_{2}$ testers always deflate the estimate of $H$ below its true value which is given by the line XY. Flowering time shows no significant departure (fig. 16) from the 


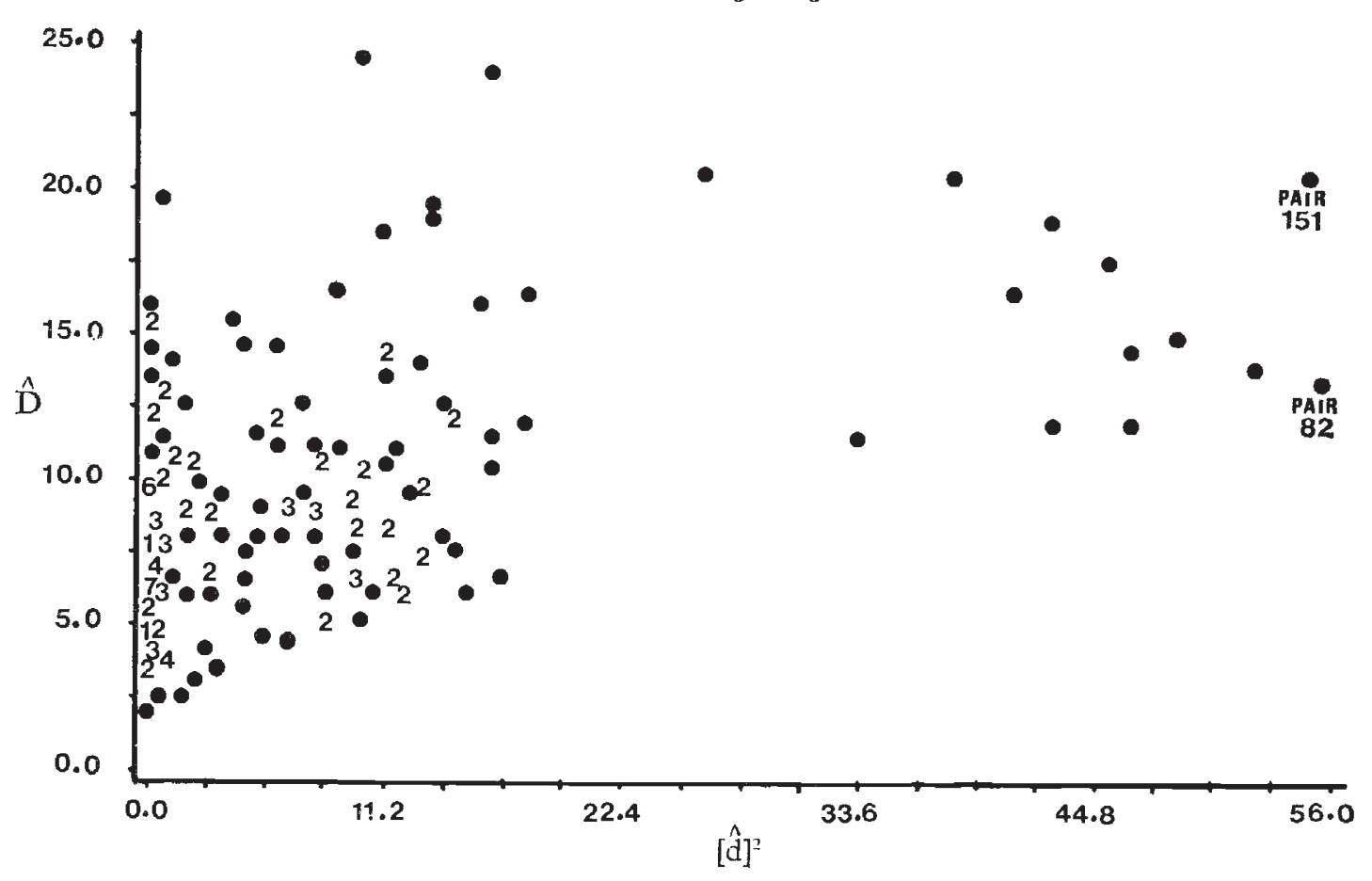

FIG. 15. $-\hat{D}$ (down) plotted against $[\hat{d}]^{2}$ (across) for flowering time.*

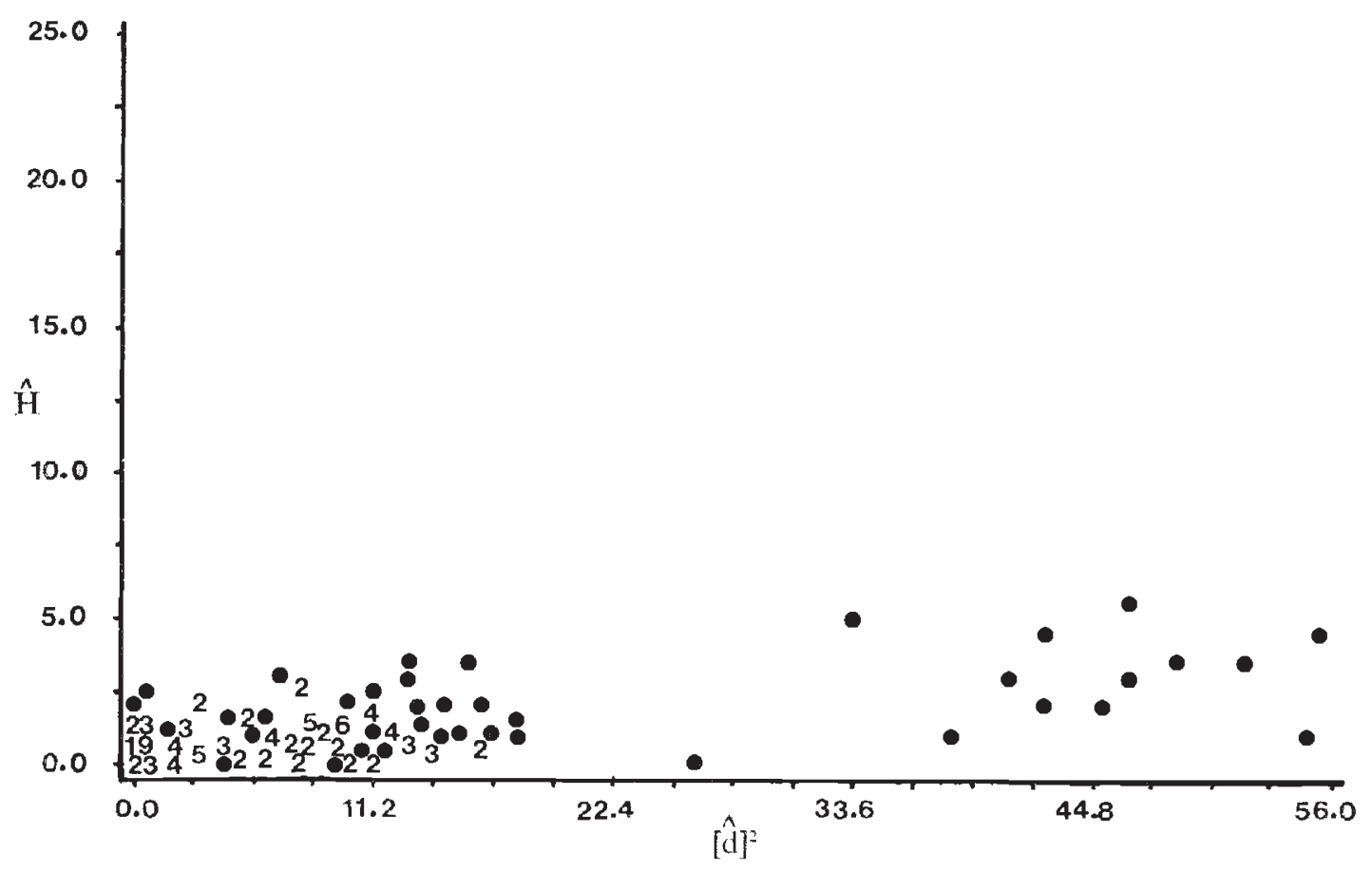

Fig. 16. $-\hat{H}$ (down) plotted against $[\hat{d}]^{2}$ (across) for flowering time.

* Footnote to figs. 15-22. Where two or more points coincide they are indicated by their frequency. 


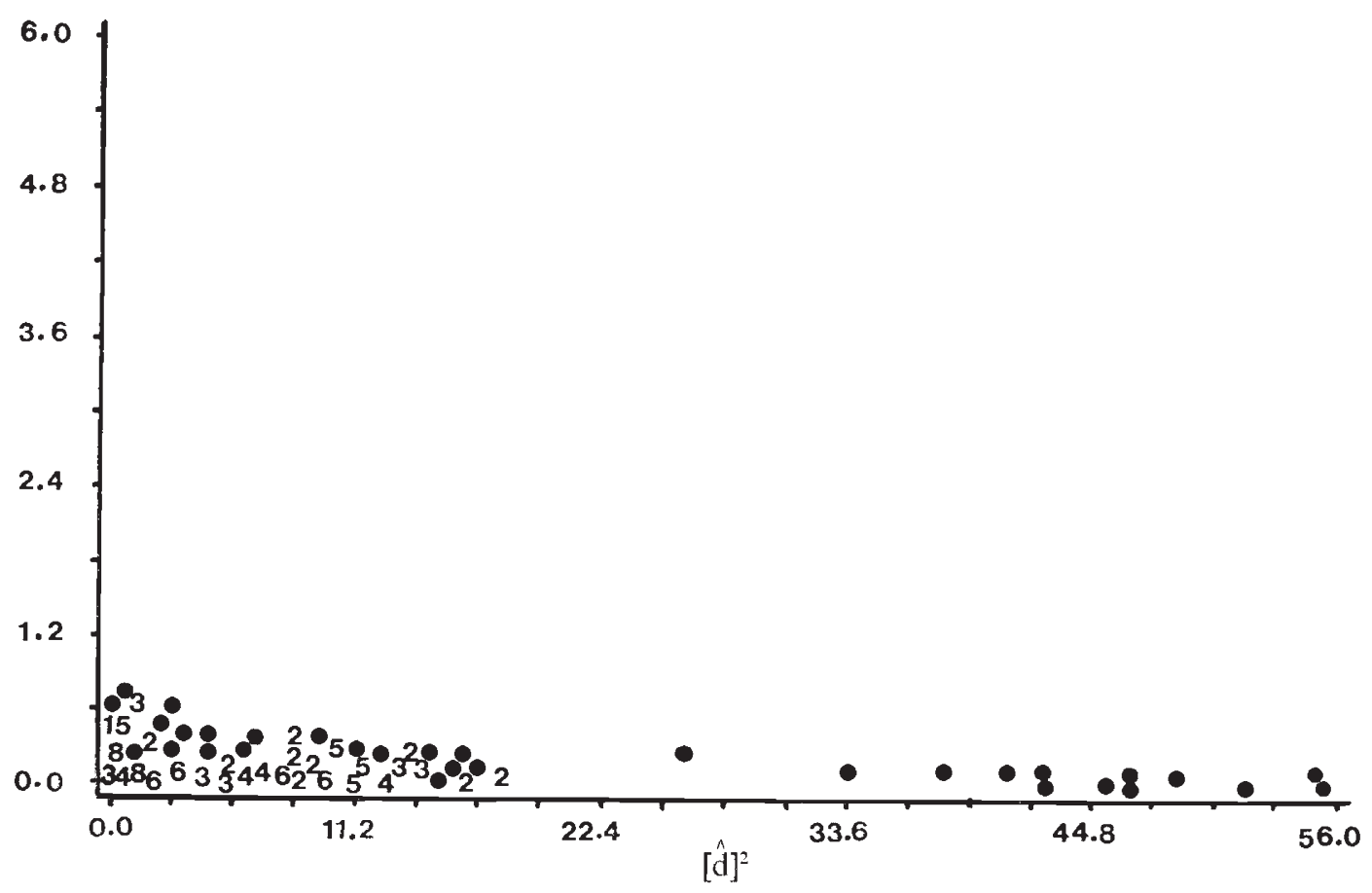

FIG. 17. - $\hat{\sigma}^{2}\left(\tilde{\mathrm{L}}_{1} i+\overline{\mathrm{L}}_{2 i}-\overline{\mathrm{P}}_{i}\right)$ (down) plotted against $[\hat{d}]^{2}$ (across) for flowering time.

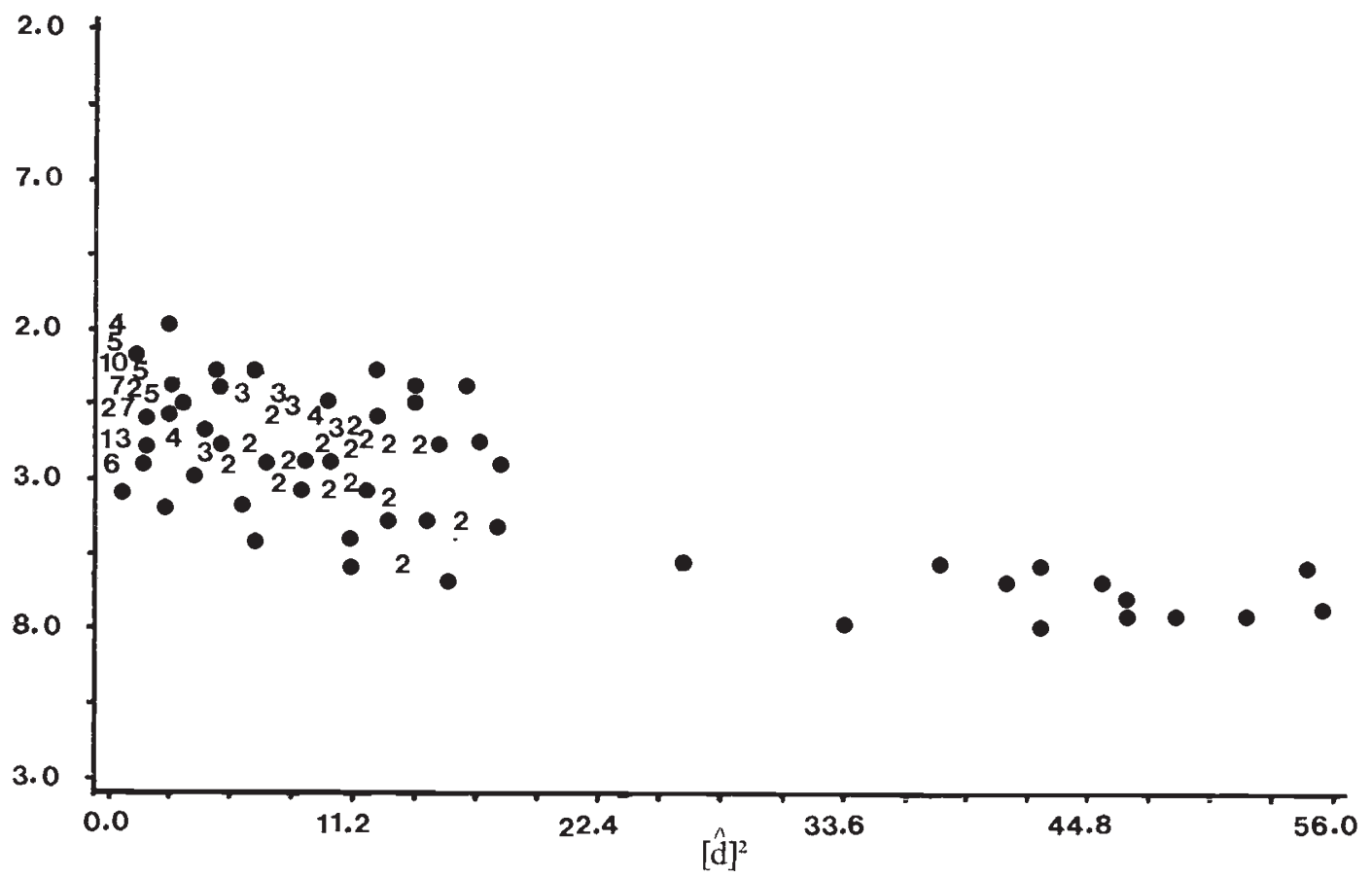

FIG. 18. $-\hat{F}$ (down) plotted against $[\hat{d}]^{2}$ (across) for flowering time. 


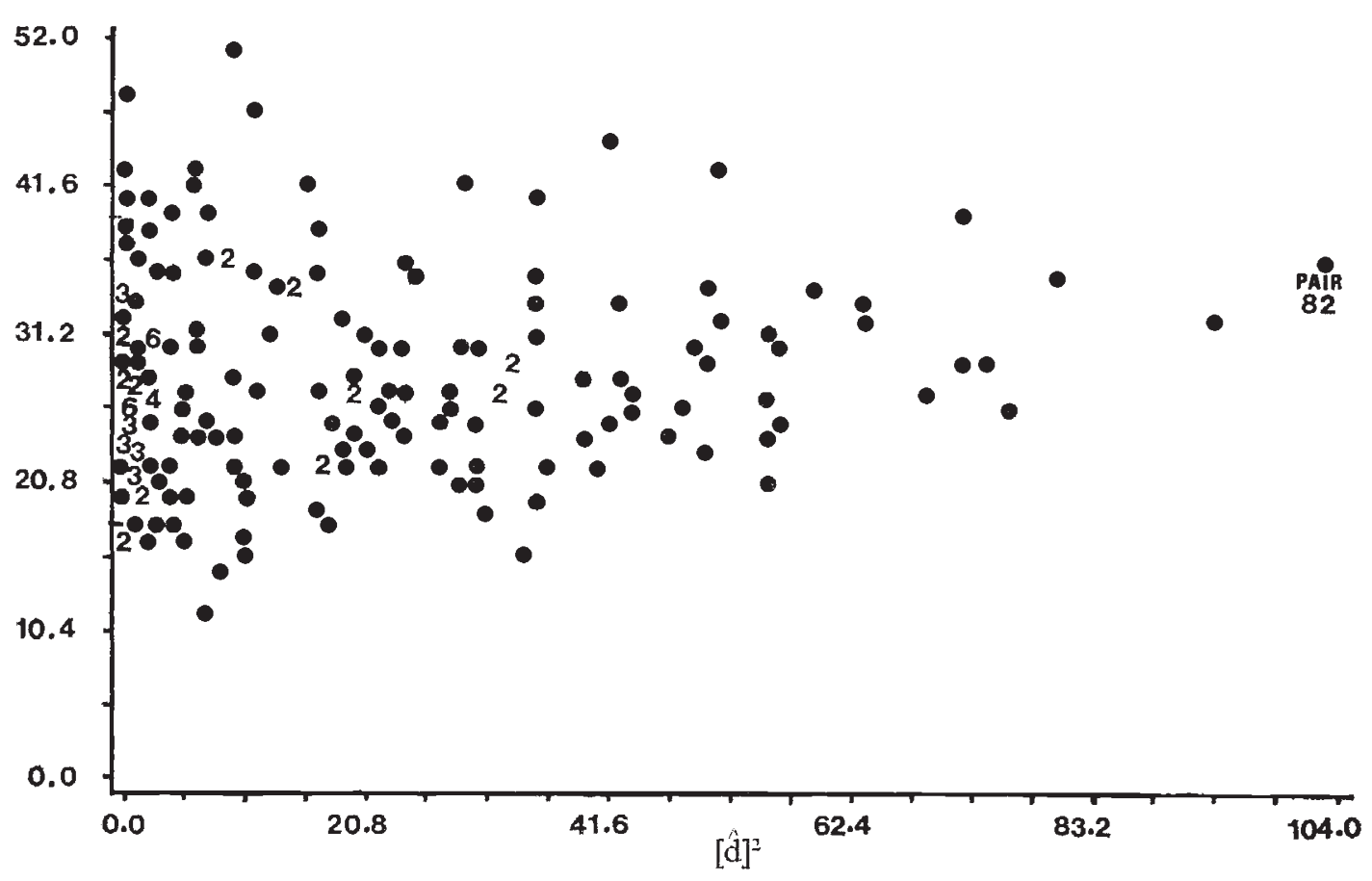

FIG. 19. $-\hat{D}$ (down) plotted against $[\hat{d}]^{2}$ (across) for plant height.

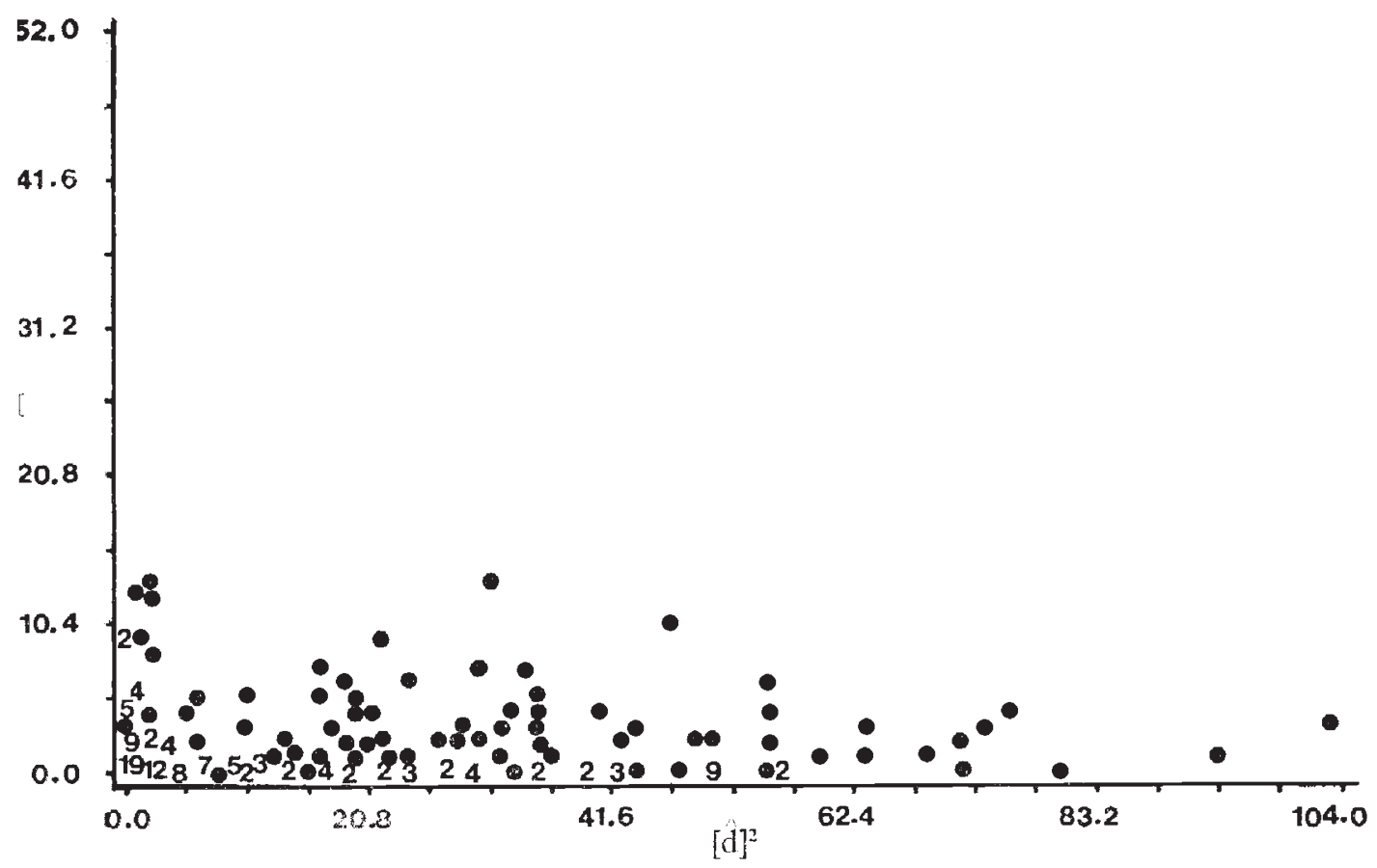

FIG. 20.- $\hat{H}$ (down) plotted against $[\hat{d}]^{2}$ (across) for plant height. 


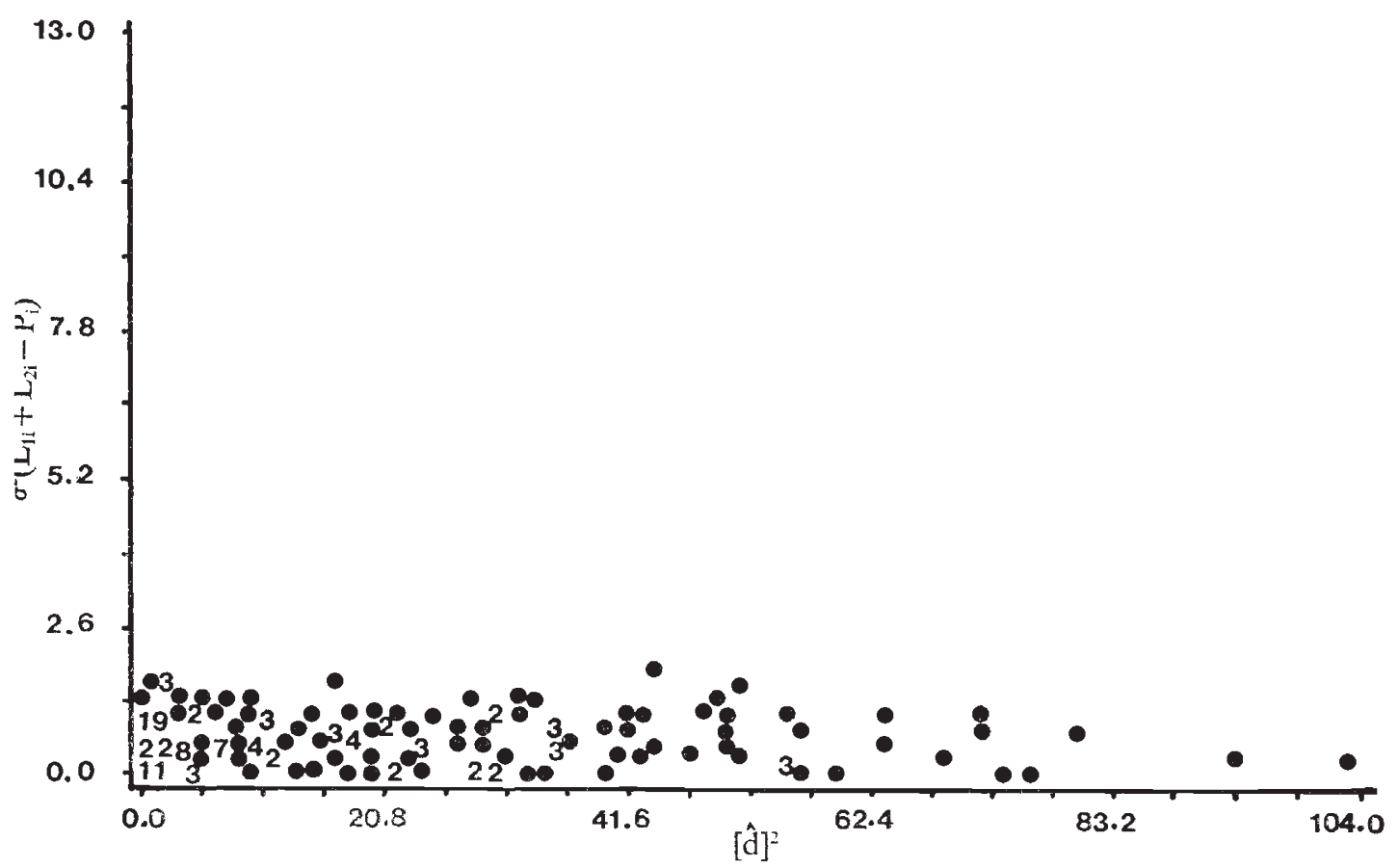

F1G. 21. - $\hat{\sigma}^{2}\left(\overline{\mathbf{L}}_{1 i}+\overline{\mathbf{L}}_{2 i}-\overrightarrow{\mathbf{P}}_{i}\right)$ (down) plotted against $[\hat{d}]^{2}$ (across) for plant height.

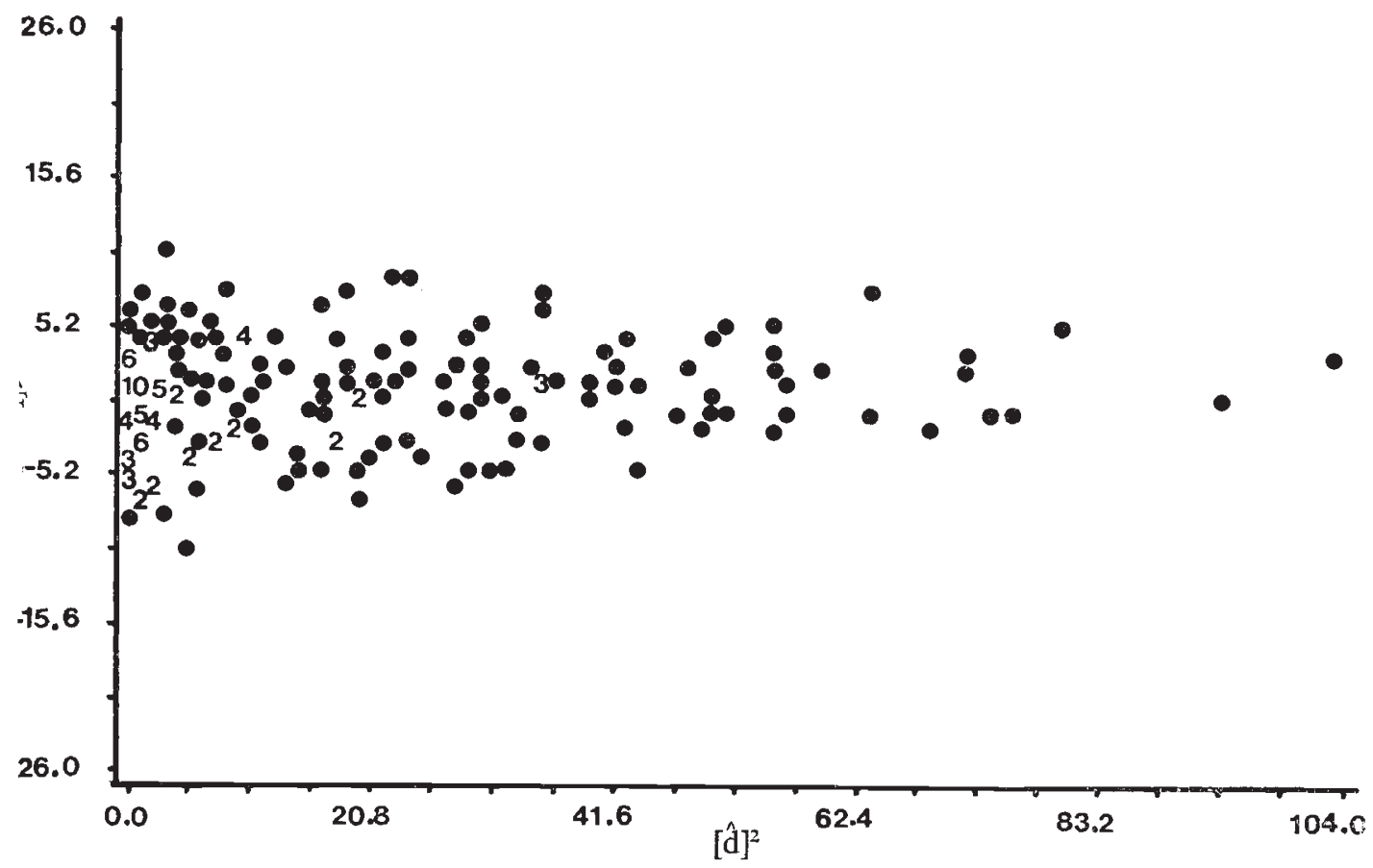

FIG. 22. $-\hat{F}$ (down) plotted against $[\hat{d}]^{2}$ (across) for plant height. 
expected relationship for partial dominance (fig. 2). Plant height (fig. 20) on the other hand, resembles more closely the distribution in fig. 9 based on partial but unequal dominance at different loci. Furthermore, the highest estimate of $H$ does not differ significantly from the unbiased estimate which is as expected.

\section{(iii) Relationship between $\sigma^{2}\left(\bar{L}_{1 \mathrm{i}}+\bar{L}_{2 \mathrm{i}}-\bar{P}_{\mathrm{i}}\right)$ and $[\mathrm{d}]^{2}$}

The observed relationships for flowering time and plant height presented in figs. 17 and 21, respectively, agree closely with the expectations in figs. 6 and 13 based solely upon the distribution of the biases resulting from common alleles. No expectations have been included for the presence of epistasis which also contributes to this variance although a low level is suspected for flowering time (see Section 4).

\section{(iv) Relationship between $\mathrm{F}$ and $[\mathrm{d}]^{2}$}

The distribution for flowering time in fig. 18 is that expected (fig. 7) for dominance of the alleles for earliness. Of the 190 pairs, $\hat{F}$ was significant and positive for 2 and negative for 47 at $\mathrm{P}<0.05$. This is consistent with mainly directional dominance for earliness. The observed relationship for final height (fig. 22) follows more closely that expected for a low level of ambidirectional and unequal dominance (fig. 14). Of the 12 significant $\hat{F}$ values 9 were positive and 3 negative.

These $F$ 's can also be estimated from the $W r_{i}+V r_{i}$ of the Jinks and Hayman analysis of a diallel (see Mather and Jinks, 1971). Comparison of these two independent methods of estimation showed them to be in very close agreement for both characters.

\section{Discussion}

These investigations have confirmed that the genetical information obtained from the simplified triple test-cross analysis becomes increasingly biased the more alleles the testers have in common. The test for the presence of epistasis may be positive when no epistasis is present. For example, this test was positive for 80 and 95 cases out of a total of 190 triple testcrosses for flowering time and plant height, respectively even though there is little or no evidence of the presence of epistasis. For flowering time only one triple test-cross using extreme testers (number 151) showed a failure of the simple model which is difficult to attribute to common alleles. The $W r, V r$ test in the diallel analysis (see Jinks et al., 1969) and generations derived from the $F_{2}$ of an initial cross between V1 and V5 have shown the presence of a low level of a duplicate type of gene interaction but the results have been inconsistent over seasons (Jinks and Perkins, 1969), because of the effect of genotype-environmental interactions and differences in the sensitivities of different methods in detecting relatively low levels of epistasis. We cannot, therefore, rule out the possibility of a low level of epistasis for flowering time which was not detected in pair 82 .

The distribution of biases in the estimates of additive, dominance and $F$ components have been described and observed. The additive component, will always be unbiased and the test for epistasis will be reliable if dominance 
is absent. If it is present, however, and the pure-breeding testers do not differ at all loci we may wrongly conclude that epistasis is present when it is not; we may underestimate or overestimate the additive component and underestimate the dominance and $F$ components. Consequently we may make the wrong deductions about the genetical architecture and hence make the wrong predictions about the consequences of natural or artificial selection. The choice of appropriate testers for a simplified triple test-cross is, therefore, crucial. It is recommended that the testers be drawn from the population which is under investigation. Once ideal $L_{1}$ and $L_{2}$ testers for any population have been obtained these will only need to be replaced if inbred lines incorporating allelic differences at additional loci are introduced into the population.

The ideal, genetically diverse pairs of testers may be provided by a number of combinations. For example, for three loci $A A B B C C$ and aabbcc, $A A B B c c$ and $a a b b C C, A A b b C C$ and $a a B B c c$, and $A A b b c c$ and $a a B B C C$ are equally suitable pairs. Of these, however, only the completely associated pair $A A B B C G$ and $a a b b c c$ because they are also the most phenotypically diverse are easily recognisable as suitable testers without extensive breeding tests. For any suitable pair of genetically diverse testers the expectations of the additive and dominance components of variation are the same. The expectations for the epistatic and $F$ component, however, differ with the degree of association of the genes and this must be allowed for in interpreting the results of the analyses.

Acknowledgments. - This research was carried out while one of us (D. S. V.) was financially supported by the Association of Commonweal th Universities.

\section{REFERENGES}

JINks, J. L. 1956. The $\mathrm{F}_{2}$ and backcross generations from a set of diallel crosses. Heredity, $10,1-30$.

JINKs, J. L., AND PERKINS, J. M. 1969. The detection of linked epistatic genes for a metrical trait. Heredity, 24, 465-475.

JINKS, J. L., PERkINS, J. M., AND BREESE, E. L. 1969. A general method of detecting additive, dominance and epistatic variation for metrical traits. II. Application to inbred lines. Heredity, 24, 45-57.

KEARSEY, M. J., AND JiNKs, J. L. 1968. A general method of detecting additive, dominance and epistatic variation for metrical traits. I. Theory. Heredity, 22, 97-103.

MATHER, K., AND JINKs, J. L. 1971. Biometrical Genetics, 2nd edition. Chapman and Hall, London. 